\title{
From Random Telegraph to Gaussian Stochastic Noises: Decoherence and Spectral Diffusion in a Semiconductor Quantum Dot
}

\author{
A. Berthelot,${ }^{1,2,3}$ C. Voisin, ${ }^{1,2}$ C. Delalande, ${ }^{1,2}$ Ph. Roussignol, ${ }^{1,2}$ \\ R. Ferreira, ${ }^{1,2}$ and G. Cassabois ${ }^{1,2,4}$ \\ ${ }^{1}$ Laboratoire Pierre Aigrain, Ecole Normale Supérieure, 24 rue Lhomond, 75231 Paris Cedex 5, France \\ ${ }^{2}$ CNRS UMR8551, Université Pierre et Marie Curie, 24 rue Lhomond, 75005 Paris, France \\ ${ }^{3}$ Laboratoire de Physico-Chimie des Matériaux Luminescents, Université Lyon 1, Université de Lyon, \\ Campus de la Doua, 69622 Villeurbanne, France \\ ${ }^{4}$ Université Montpellier 2, CC 074, 34095 Montpellier Cedex 5, France
}

Correspondence should be addressed to G. Cassabois, guillaume.cassabois@lpa.ens.fr

Received 21 September 2009; Accepted 29 December 2009

Academic Editor: Shao-Ming Fei

Copyright (C 2010 A. Berthelot et al. This is an open access article distributed under the Creative Commons Attribution License, which permits unrestricted use, distribution, and reproduction in any medium, provided the original work is properly cited.

We present a general theoretical description of the extrinsic dephasing mechanism of spectral diffusion that dominates the decoherence dynamics in semiconductor quantum dots at low temperature. We discuss the limits of random telegraph and Gaussian stochastic noises and show that the combination of both approaches in the framework of the pre-Gaussian noise theory allows a quantitative interpretation of high-resolution experiments in single semiconductor quantum dots. We emphasize the generality and the versatility of our model where the inclusion of asymmetric jump processes appears as an essential extension for the understanding of semiconductor quantum dot physics.

\section{Introduction}

Decoherence is one of the fundamental limitations in quantum information science, and the understanding and the control of its dynamics appears as a crucial issue for the development of quantum information devices. Various systems are being investigated in condensed matter physics because of their potential in terms of integrability and scalability, such as superconducting circuits [1], edge states in the Fractional Quantum Hall effect [2], and semiconductor quantum dots (QDs) [3]. In the latter case, the development of spatially-resolved optical spectroscopy has allowed the implementation of quantum information schemes in single QDs. Single photon emission [4], photon coalescence [5], 
and quantum gate implementation [6] have successfully been demonstrated with an efficiency intrinsically limited by the decoherence due to the coupling of the QD with its environment. In this review, we discuss the extrinsic dephasing mechanism of spectral diffusion that dominates the QD decoherence at low temperature. We present a theoretical model in the framework of the pre-Gaussian noise theory where random telegraphs and Gaussian stochastic noises are combined. We illustrate the generality of our model with quantitative interpretations of the different signatures of spectral diffusion in single QDs.

\section{Theory}

\subsection{Spectral Diffusion in Semiconductor Quantum Dots}

At low temperature, the decoherence in QDs is mainly determined by the phenomenon of spectral diffusion. In fact, photoluminescence experiments in single QDs have revealed the extrinsic influence of the solid matrix that generates fluctuating electric fields and shifts the QD line through the quantum confined Stark effect. This so-called spectral diffusion phenomenon was interpreted as due to carriers randomly trapped in defects, impurities in the QD vicinity [7-9]. Given the time-resolution imposed by the detector integration time, the emission spectrum can be analyzed by considering two different classes of random events. The first one is associated to rare and strongly-shifting processes that result in abrupt jumps of the QD emission energy, that is, spectral discontinuities in the timeevolution of the photoluminescence spectrum. In the case of colloidal QDs or nanocrystals, these events may also inhibit the emission and the corresponding blinking effect [7] is a severe restriction to the realization of efficient single QD-devices based on this type of nanostructures. The second type of events is related to frequent and weakly-shifting processes that lead, within the detector integration time, to a single line with a width that is larger than the radiative limit and that is determined by the statistical distribution of the small energy shifts. However, these different phenomenologies are usually separately and partially addressed in the theoretical descriptions developed in colloidal and epitaxial QDs. We present in this review a general model that reaches a full comprehensive description of spectral diffusion in semiconductor QDs and that provides a flexible framework for the quantitative interpretation of experimental data in semiconductor QDs. In particular, we emphasize that our theoretical model takes into account a specific and original point, namely the dynamical asymmetry of the spectral jump processes that is not discussed in previous theoretical work [10] while being of fundamental importance in the physics of semiconductor nanostructures [11].

Our model is based on a Markov chain composed of an arbitrary number of $N$ independent random telegraphs. A random telegraph is a two-state jump process that corresponds to a discrete spectral shift $\delta \omega= \pm \Omega / 2$ of the optical line. The transition from the upper (lower) state to the lower (upper) one occurs with a probability $d t / \tau_{\downarrow}\left(d t / \tau_{\uparrow}\right)$ in the time interval $d t$ and induces a spectral jump $-\Omega / 2(+\Omega / 2)$, as sketched in Figure 1 . In the context of spectral diffusion in QDs, the upper (lower) state corresponds to an empty (occupied) defect, $\tau_{\downarrow}\left(\tau_{\uparrow}\right)$ to the capture (escape) time of one carrier in the defect, and $\Omega$ to the Stark shift of the QD line due to the electric field created by the charge carrier in the defect located in the QD vicinity. 


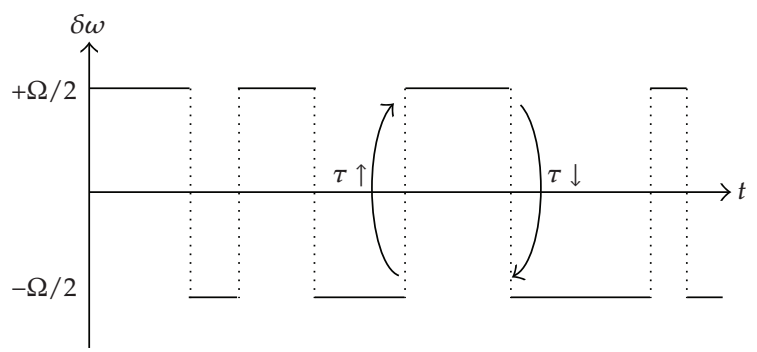

Figure 1: Frequency fluctuation of the QD emission line as a function of time in the presence of a single random telegraph. $\Omega$ is the Stark shift of the QD line due to the electric field created by the charge carrier in the defect located in the QD vicinity. $\tau_{\downarrow}$ and $\tau_{\uparrow}$ are the characteristic time constants of the jump processes. reads

In the presence of $N$ random telegraphs, the modulated frequency $\omega(t)$ of the QD line

$$
\omega(t)=\omega_{0}+\delta \omega(t)
$$

where $\omega_{0}$ is the time average of the QD frequency and $\delta \omega(t)$ its fluctuating part given by

$$
\delta \omega(t)=\sum_{i=1}^{N} \delta \omega_{i}(t)
$$

where $\delta \omega_{i}$ denotes the frequency fluctuation caused by a single random telegraph.

According to the Wiener-Khintchine theorem, the optical spectrum is given by the Fourier transform of the electric field autocorrelation function which is proportional to the so-called relaxation function $\phi_{N}(t)$

$$
\left\langle E^{*}\left(t+t^{\prime}\right) E(t)\right\rangle=\left|E_{0}\right|^{2} \exp \left(i \omega_{0} t^{\prime}\right) \phi_{N}\left(t^{\prime}\right),
$$

where $\phi_{N}(t)$ reads [12]

$$
\phi_{N}(t)=\left\langle\exp \left(i \int_{0}^{t} \delta \omega(u) d u\right)\right\rangle,
$$

(where $\langle\cdots\rangle$ denotes the average over different configurations) so that the optical spectrum is obtained by the Fourier transform of the relaxation function:

$$
I\left(\omega-\omega_{0}\right)=\int_{-\infty}^{+\infty} \exp \left(-i\left(\omega-\omega_{0}\right) t\right) \phi_{N}(t) d t
$$


By using (2.2) and the assumption of uncorrelation for the different random telegraphs [10], the total relaxation function $\phi_{N}(t)$ is rewritten as

$$
\phi_{N}(t)=\left\langle\prod_{i=1}^{N} \exp \left(i \int_{0}^{t} \delta \omega_{i}(u) d u\right)\right\rangle=\prod_{i=1}^{N} \phi_{1_{i}}(t),
$$

where $\phi_{1_{i}}(t)$ denotes the particular relaxation function of the $i$ th single telegraph, which is given by

$$
\phi_{1_{i}}(t)=\left\langle\exp \left(i \int_{0}^{t} \delta \omega_{i}(u) d u\right)\right\rangle
$$

\subsection{Random Telegraph Noise}

The intensity spectrum in the presence of frequency fluctuation due to Markovian processes was addressed by Kubo in his seminal paper on the stochastic theory of lineshape where the general resolution of the Chapman-Kolmogorov equation was presented [12]. The stochastic theory of lineshape was further completed by Wodkiewicz and coworkers in their preGaussian noise theory [10]. They made the link between random telegraphs and Gaussian stochastic processes by calculating the relaxation function $\phi_{N}(t)=\left[\phi_{1}(t)\right]^{N}$ for a given number of $N$ identical, uncorrelated random telegraphs, and by then expanding their treatment to the limit $N \rightarrow \infty$. However, this model is limited to the case of symmetric jump processes $\left(\tau_{\downarrow}=\tau_{\uparrow}\right.$; see Figure 1$)$.

In the prospect of a more general theory that would catch the specific physics of semiconductor nanostructures, we have extended the former model to the case of asymmetric two-state jump processes and derived the expression of the relaxation function $\phi_{1}(t)$ when $\tau_{\uparrow} \neq \tau_{\downarrow}$. Following the guidelines of the Kubo theory, we find for the generalized analytical expression of the relaxation function $\phi_{1}(t)$ :

$$
\phi_{1}(t)=G_{+1}(t)-G_{-1}(t)
$$

with

$$
G_{\varepsilon}(t)=\frac{1+\varepsilon Y+i \eta X}{2 Y} \exp \left[-(1-\varepsilon Y) \frac{|t|}{2 \tau_{c}}\right]
$$

where the correlation time $\tau_{c}$ is given by $1 / \tau_{c}=1 / \tau_{\uparrow}+1 / \tau_{\downarrow}, \eta=\left(\tau_{\uparrow}-\tau_{\downarrow}\right) /\left(\tau_{\uparrow}+\tau_{\downarrow}\right)$ characterizes the intensity asymmetry in the emission spectrum, $X=\Omega \tau_{c}$, and $Y$ is given by $Y^{2}=1-X^{2}+$ 2i $\chi X$ with the condition $\mathfrak{R}(Y) \geq 0$. In the simple case of symmetric jump processes $(\eta=0)$, one easily recovers for $\phi_{1}(t)$ the expression given in [10].

If $\Omega \tau_{c} \gg 1$, the system is in the so-called slow modulation limit. Let us first examine the simple case $\eta=0$ corresponding to symmetric jump processes, where $\phi_{1}(t)$ is simplified in

$$
\phi_{1}(t) \sim \exp \left(-\frac{|t|}{2 \tau_{c}}\right) \cos \left(\frac{\Omega t}{2}\right)
$$




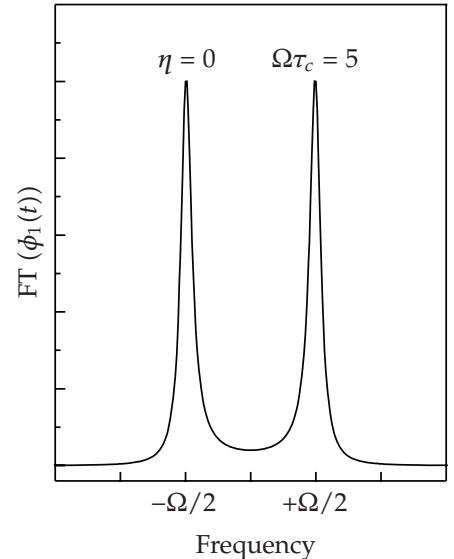

(a)

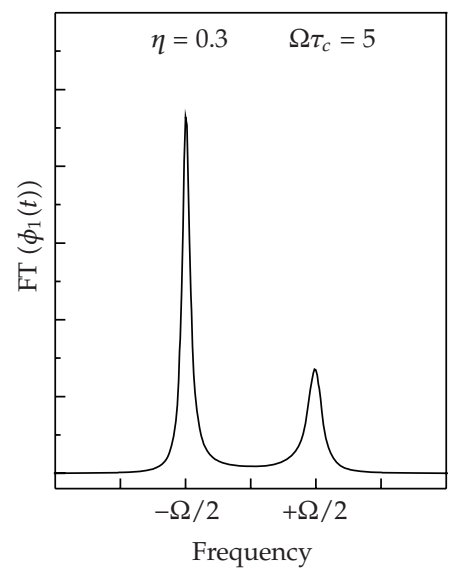

(d)

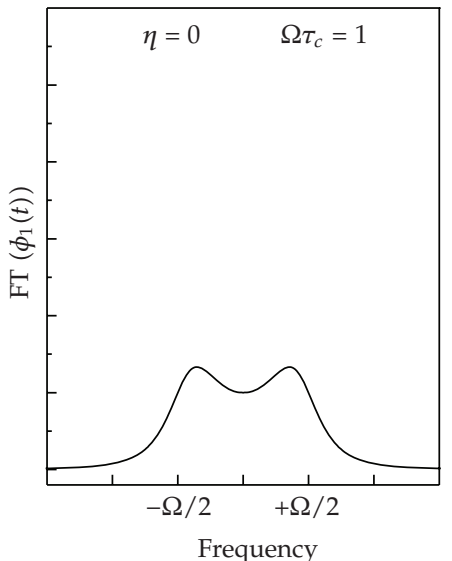

(b)

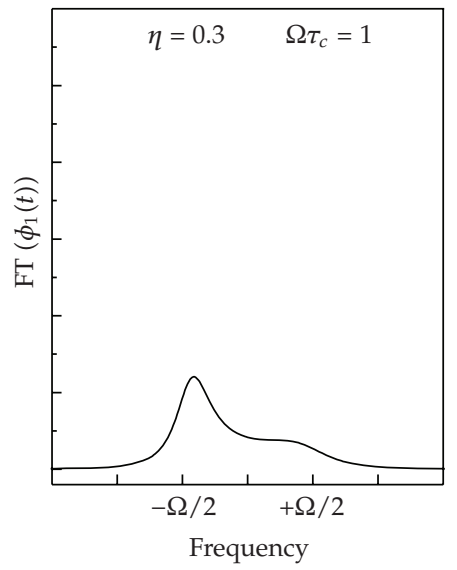

(e)

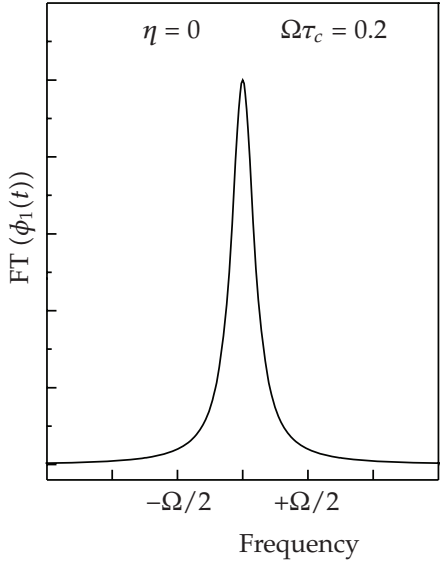

(c)

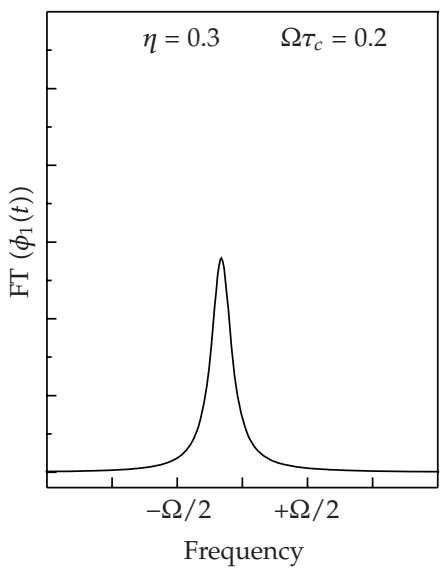

(f)

Figure 2: Optical spectrum in the presence of a single random telegraph: the Fourier transform of the relaxation function $\phi_{1}(t)$ is plotted for symmetric $(\eta=0)$ and asymmetric $(\eta=0.3)$ jump processes for three different values of $\Omega \tau_{c}=5,1$, and 0.2 .

By taking the Fourier transform of (2.10), one gets for the optical spectrum a symmetric doublet structure, with two lines split by $\Omega$ and a full width at half maximum $1 / \tau_{c}$ (Figure 2(a)). In other words, when $\Omega \tau_{c} \gg 1$, the optical spectrum reflects the statistical distribution corresponding to Figure 1, with a line-broadening given by the fluctuating rate $1 / \tau_{c}$. For asymmetric jump processes, the optical spectrum exhibits an asymmetric doublet where the ratio of the integrated intensity of the two separate lines is given by $(1-\eta) /(1+\eta)$ (Figure 2(d)).

On the contrary, in the fast modulation limit or motional narrowing regime $\left(\Omega \tau_{c} \ll 1\right)$, the reduction of the accumulated phase induces the spectral coalescence of the two lines and the doublet structure disappears [12]. In the simple case of symmetric jump processes $(\eta=0)$, the relaxation function $\phi_{1}(t)$ now reads

$$
\phi_{1}(t) \sim \exp \left(-\frac{\Omega^{2} \tau_{c}|t|}{4}\right)
$$


that corresponds in the Fourier domain to a single Lorentzian line with a full width at half maximum given by $\Omega^{2} \tau_{c} / 2$. As can be seen in Figures 2(a), 2(b), and 2(c), when $\Omega \tau_{c}$ decreases from 5 (slow modulation) to 0.2 (fast modulation), the spectrum transforms from a doublet with a splitting $\Omega$ to a single Lorentzian line with a width given by the product $\Omega^{*}\left(\Omega \tau_{c} / 2\right)$, thus meaning that the shorter the correlation time, the narrower the line compared to the splitting $\Omega$. For $\eta \neq 0$, the coalescence of the asymmetric doublet leads to a single Lorentzian line that is spectrally detuned (Figure 2(f)) towards the energy of the more intense line (Figure 2(d)).

\subsection{Gaussian Stochastic Noise}

We now switch to the more realistic situation where a large number of random telegraphs contribute to the spectral diffusion of a QD line. For $N$ independent, identical two-state jump processes, the relaxation function $\phi_{N}(t)$ reads $\left[\phi_{1}(t)\right]^{N}$, and when $N \gg 1$, Wódkiewicz et al. showed that the relaxation function $\phi_{N}(t)$ converges to the one obtained for a Gaussian noise $\phi_{\mathrm{G}_{N}}(t)[10]$ :

$$
\phi_{G_{N}}(t)=\exp \left[-\Sigma^{2} \tau_{c}^{2}\left(\exp \left(-\frac{|t|}{\tau_{c}}\right)+\frac{|t|}{\tau_{c}}-1\right)\right]
$$

which is a consequence of the central limit theorem in the context of spectral noise. The parameter $\Sigma$ in (2.12) is the standard deviation of the QD frequency, and in the simple case of symmetric jump processes $\left(\tau_{\uparrow}=\tau_{\downarrow}\right), \Sigma$ is given by $\sqrt{N} \Omega / 2$ [10].

In order to complete our generalization of the spectral diffusion theory and address the case of Gaussian stochastic processes with asymmetric jump events, we have derived the general expression of $\Sigma$ when $\tau_{\uparrow} \neq \tau_{\downarrow}$, and we find for the frequency modulation amplitude $\Sigma$ [11]:

$$
\Sigma=\frac{\sqrt{N} \Omega}{\sqrt{\tau_{\uparrow} / \tau_{\downarrow}}+\sqrt{\tau_{\downarrow} / \tau_{\uparrow}}}
$$

which reaches its maximum value $\Sigma_{s}=\sqrt{N} \Omega / 2$ when $\tau_{\uparrow}=\tau_{\downarrow}$ and vanishes in the limit of strong asymmetric jump processes $\left(\tau_{\uparrow} \gg \tau_{\downarrow}\right.$ or $\left.\tau_{\uparrow} \ll \tau_{\downarrow}\right)$, as shown in Figure 3 .

If $\Sigma \tau_{c} \gg 1$, the system is in the slow modulation limit, in analogy to the single random telegraph phenomenology described above. The relaxation function dynamics are approximated by the Gaussian function

$$
\phi_{G_{N}}(t) \sim \exp \left(-\frac{\Sigma^{2} t^{2}}{2}\right)
$$

and, in the Fourier domain, the optical spectrum has a Gaussian profile that reflects the Gaussian distribution law of the spectral noise. The full width at half maximum depends solely on the frequency modulation amplitude and is given by $2 \sqrt{2 \ln 2} \Sigma$. 


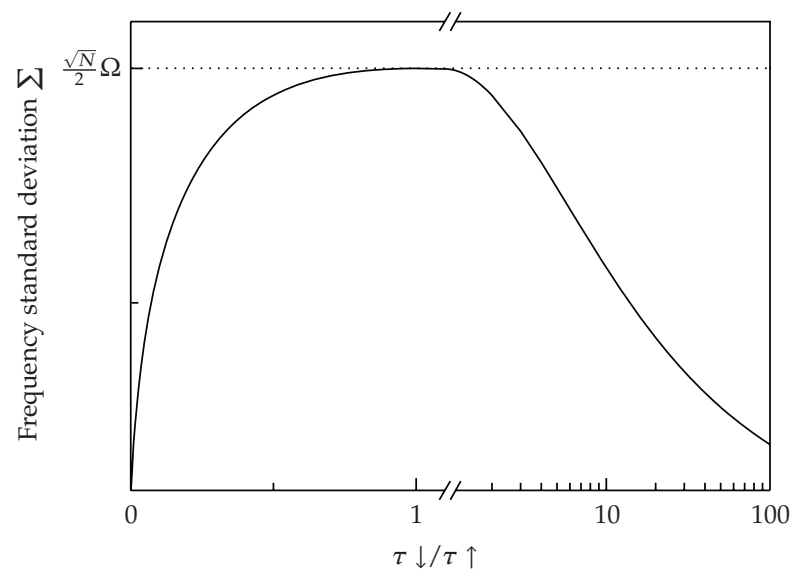

Figure 3: Standard deviation $\Sigma$ of the frequency versus ratio of the jump processes characteristic times $\tau_{\downarrow} / \tau_{\uparrow}$. The dotted line indicates the maximum value of the standard deviation $\Sigma_{s}=\sqrt{N} \Omega / 2$ that is reached for symmetric jump processes $\tau_{\downarrow}=\tau_{\uparrow}$.

In the fast modulation limit $\Sigma \tau_{c} \ll 1$, the relaxation function dynamics are strongly modified and now correspond to an exponential decay:

$$
\phi_{G_{N}}(t) \sim \exp \left(-\Sigma^{2} \tau_{c}|t|\right)
$$

which is the analog for Gaussian stochastic processes of the motional narrowing effect described above for a random telegraph. The Fourier transform of (2.15) is a Lorentzian line with a width of $2 \Sigma^{2} \tau_{c}$, that is, the shorter the correlation time the narrower the optical spectrum.

We will show now that the common configuration of Gaussian stochastic processes displays a rich and original phenomenology in the case of asymmetric jump processes $\tau_{\uparrow} \neq \tau_{\downarrow}$. In particular, we analyze the crossover from the slow modulation limit to the fast modulation one and show that the transition to motional narrowing may be completely different in the two cases $\tau_{\uparrow}=\tau_{\downarrow}$ and $\tau_{\uparrow} \neq \tau_{\downarrow}$.

For symmetric jump processes, the frequency modulation amplitude is constant with a value given by $\sqrt{N} \Omega / 2$. As a matter of fact, the transition from $\Sigma \tau_{c} \gg 1$ to $\Sigma \tau_{c} \ll 1$ implies that $\tau_{c}$ decreases, and thus that $\tau_{\uparrow}$ decreases since $\tau_{c}=\tau_{\uparrow} / 2$. In other words, while $\tau_{\uparrow}$ decreases, the line-profile changes from Gaussian to Lorentzian, and the linewidth decreases from $2 \sqrt{2 \ln 2} \Sigma$ to $2 \Sigma^{2} \tau_{c}$. This case corresponds to the standard phenomenology of motional narrowing, which was originally experimentally observed in nuclear magnetic resonance where $\tau_{\uparrow}$ decreases as a function of temperature because of the activation of the nuclei motion [12].

However, for asymmetric jump processes, the situation is totally different since the frequency modulation is no longer constant and strongly depends on the ratio $\tau_{\downarrow} / \tau_{\uparrow}((2.13)$ and Figure 3). Let us take the case of a strong asymmetry $\tau_{\downarrow} \ll \tau_{\uparrow}$ and assume for the sake of clarity that $\tau_{\downarrow}$ is furthermore constant (we will show below an example of this phenomenology in the particular case of QDs). In this case, we see that the correlation time has now a constant value given by $\tau_{\downarrow}$ while $\Sigma$ varies, that is the opposite situation as the one depicted above for symmetric jump processes. Consequently, when $\tau_{\uparrow}$ decreases, we 
get the reverse transition from the fast to the slow modulation limit. This peculiarity of asymmetric jump processes is a fundamental originality of QDs physics compared to nuclear magnetic resonance and it explains why motional narrowing appears in very different situations as shown below.

We illustrate the generality and the versatility of our model by several examples based on experimental data recorded by means of high-resolution Fourier-transform spectroscopy in single QDs.

\section{Experiments}

We present measurements performed in self-assembled InAs/GaAs QDs grown by molecular beam epitaxy in the Stranski-Krastanow mode, and micro-photoluminescence measurements under nonresonant excitation are performed in the far field using the experimental setup described in [13]. In order to accurately characterize the QD emission spectrum, the photoluminescence signal arising from a single QD is analyzed by means of Fouriertransform spectroscopy. Our technique allows a high-resolution sampling of the Fouriertransform of the spectrum on typically thousands of points. This value is by two orders of magnitude larger than the average number of illuminated pixels in a charge-coupled device in the case of a multichannel detection in the spectral domain. The Fourier-transform technique is implemented in the detection part of the setup where the photoluminescence signal passes through a Michelson interferometer placed in front of a grating spectrometer [13]. The signal is detected by a low noise Si-based photon counting module. With a translation stage, we vary the time $t$ for propagation in one arm of the interferometer and record interferograms of the photoluminescence emission $I(t)=I_{0}\left(1+C(t) \cos \left(\omega_{0} t\right)\right)$, where $I_{0}$ is the average photoluminescence signal intensity, $\omega_{0}$ the central detection frequency, and $C(t)$ the interference contrast which corresponds to the modulus of the Fourier transform of the optical spectrum, that is, with the notation above $C(t)=\left|\phi_{N}(t)\right|$. The implementation of this technique in single QD spectroscopy allows a precise determination of both width and shape of the emission line in order to accurately study the spectral diffusion phenomenon and the related QD decoherence.

\subsection{Singular Random Telegraph}

We first present the experimental evidence for a random telegraph noise where the asymmetry of the jump processes is tuned with a dc-voltage. This effect is observed in the emission spectrum of a single QD embedded in a field-effect heterostructure as a pronounced beating in the Fourier transform measurements. The beating visibility varies as a function of the gate voltage applied to the device, thus showing the control of the doublet asymmetry with the internal electric field.

The sample consists of a single QD embedded in the field-effect structure depicted in Figure 4. The InGaAs QDs are separated by a $25 \mathrm{~nm}$ GaAs layer from a highly n-doped GaAs substrate. The QDs are capped by $15 \mathrm{~nm}$ of GaAs, followed by a $75 \mathrm{~nm}$ AlGaAs blocking barrier and finally $60 \mathrm{~nm}$ of GaAs. After growth, a $10 \mathrm{~nm}$ thick semitransparent Ti Schottky contact (right side of Figure 4) was deposited on the top surface. In order to study single QDs, we use a $200 \mathrm{~nm}$ thick Au mask into which $400 \mathrm{~nm}$ diameter apertures are opened lithographically. 


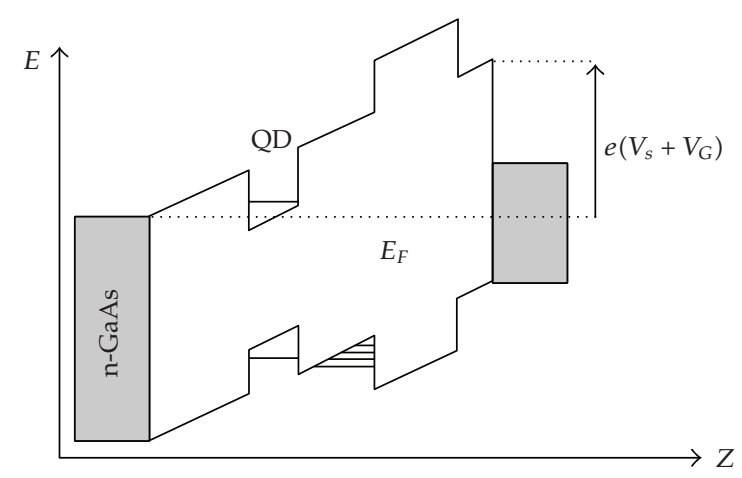

Figure 4: Schematic sketch of the heterostructure (under reverse bias) displaying the conduction and valence band edges energy along the growth direction. The applied gate voltage $V_{G}$ is superimposed on the Schottky voltage $V_{S}$ of the order of $-1.5 \mathrm{~V}$ for our structure.

In Figure 5, we display the interferogram contrasts $C(t)$ on semilogarithmic plots for the neutral exciton line at $10 \mathrm{~K}$, for the three gate voltages of $-0.24(\mathrm{a}),-0.22(\mathrm{~b})$, and $-0.2 \mathrm{~V}$ (c). We observe a strong beating, with a contrast close to unity for $-0.22 \mathrm{~V}$ (Figure $5(\mathrm{~b})$ ). This means that the photoluminescence spectrum consists, in that case, in a doublet with identical lines. From the beating period, we estimate an energy splitting $\hbar \Omega$ of the order of $44 \mu \mathrm{eV}$. By changing the linear polarization of analysis, there is no modification of the data, thus excluding any effect related to the fine-structure splitting of the neutral exciton line [14]. The dependence of the beating contrast on gate voltage, Figures 5(a) and 5(c), is a signature of the electrical control of the asymmetry of the jump processes involved in the random telegraph.

In the framework of the pre-Gaussian noise theory, we take the product $\phi_{1}(t) \phi_{G_{N}}(t)$ for the total relaxation function $\phi_{N+1}(t)$, where $\phi_{1}(t)$ and $\phi_{G_{N}}(t)$ are, respectively, given by (2.8) and (2.12). This choice means that we separate the $N+1$ independent two-state jump processes into a large number $(N \gg 1)$ of similar ones inducing a Gaussian noise, and a singular one. The latter is responsible for the strong perturbation leading to the beating and doublet structure, and the former for the broadening of each component. In Figure 5, we display in solid line the calculated contrasts $C(t)=\left|\phi_{1}(t) \phi_{G_{N}}(t)\right|$. We observe an excellent agreement thus showing that our model quantitatively reproduces the whole emission spectrum (shape and width). We fit our complete set of data as a function of voltage and temperature $\left(10 \mathrm{~K}\right.$, and $20 \mathrm{~K}, 30 \mathrm{~K}$ not shown here) by taking $\hbar \Sigma_{s}=28 \mu \mathrm{eV}, \hbar \Omega=44 \mu \mathrm{eV}$, $\tau_{\downarrow}=45 \mathrm{ps}$ for the Gaussian noise, $\tau_{\downarrow}=500 \mathrm{ps}$ for the singular random telegraph, and by only varying the escape times $\tau_{\uparrow}$ with the values indicated in Figure 5(d) for the singular random telegraph.

In Figure 5(d), we observe a systematic decrease of the escape time $\tau_{\uparrow}$ with the reverse bias, as expected for field-induced tunneling. In analogy to the thermo-ionization of deep centers [15], we take a phonon-assisted tunneling rate that is proportional to the transmission through a triangular barrier due to a static electric field [15]. We reproduce our data by taking $\tau_{\uparrow}=\tau_{\infty} \top(F)^{-1}$ where $T(F)$ is the barrier transmission given by $\exp \left(-4 \sqrt{2 m^{*} E_{i}^{3}} / 3 \hbar e F\right)$ with $F$ the electric field strength along the growth direction, $m^{*}$ the effective mass, and $E_{i}$ the effective ionization energy. We fit our data with $E_{i}^{e}=245 \mathrm{meV}$ in the case of electrons $\left(m^{*}=\right.$ $\left.0.07 m_{0}\right)$ or $E_{i}^{h}=145 \mathrm{meV}$ for holes $\left(m^{*}=0.34 m_{0}\right), \tau_{\infty}=2 \times 10^{-4} \mathrm{ps}$. The large values of $E_{i}$ suggest that the spectral diffusers are deep defects, in agreement with the recent experimental 


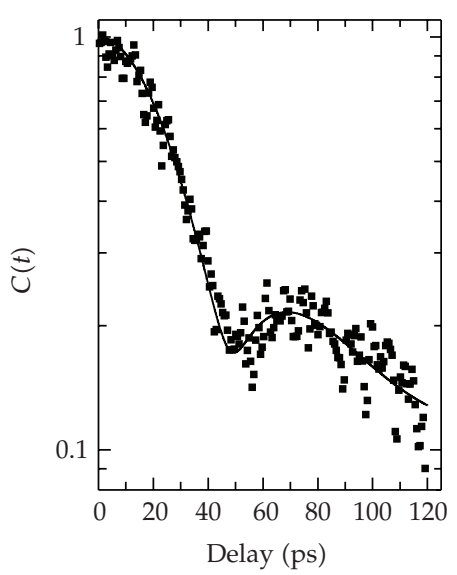

(a)

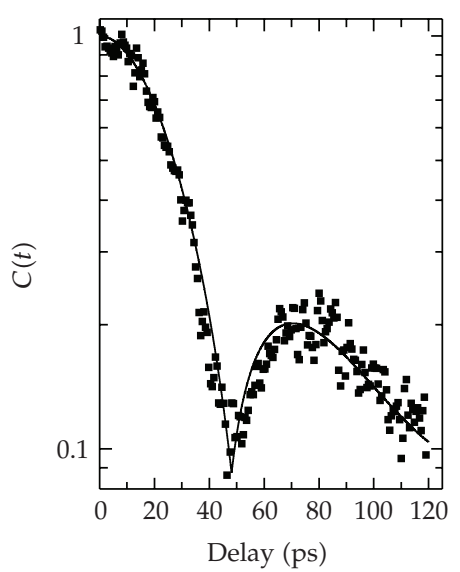

(b)

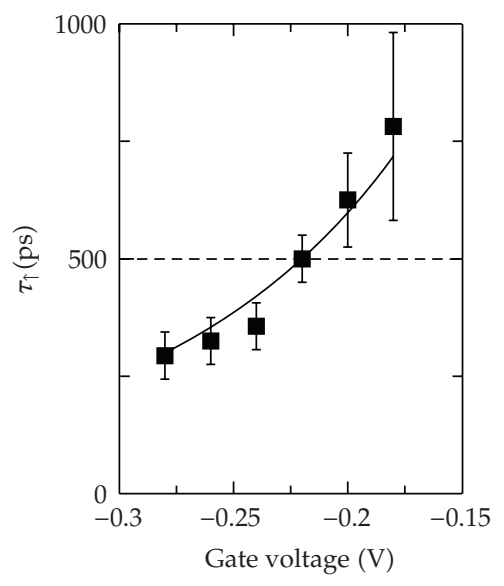

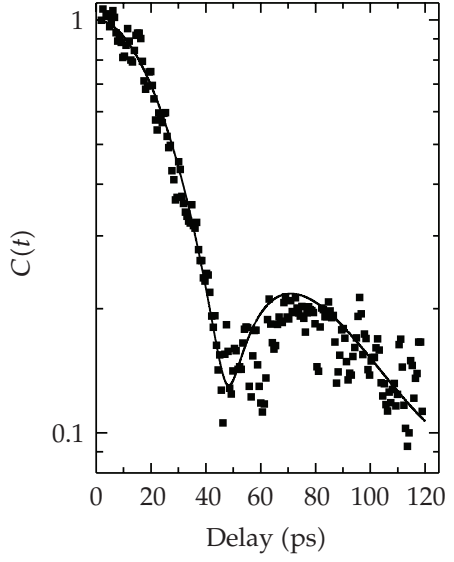

(c)

(d)

Figure 5: (a), (b), (c): Interferogram contrast $C(t)$ of the photoluminescence signal of a single InGaAs QD at $10 \mathrm{~K}$, on semilogarithmic plots, for three gate voltages: $-0.24 \mathrm{~V}(\mathrm{a}),-0.22$ (b), and $-0.2 \mathrm{~V}$. Data (squares), theoretical fits corresponding to $\left|\phi_{1}(t) \phi_{G_{N}}(t)\right|$ (solid line). (d): Tunneling time versus gate voltage. Data (symbols), theoretical fit (solid line). The horizontal line indicates the capture time $\tau_{\downarrow}=500 \mathrm{ps}$.

evidence by deep-level transient spectroscopy of the coexistence of deep levels with optically active InAs QDs [16].

In the example above, the jump processes asymmetry is tailored by means of a dc-gate voltage that controls the fluctuation dynamics of a singular random telegraph. We discuss below another example where the jump processes asymmetry is modified by varying other external experimental parameters, but in the case of a Gaussian stochastic noise.

\subsection{Crossover from the Fast to Slow Modulation Limit}

We present here the experimental evidence for a crossover from the fast to the slow modulation limits in the case of a Gaussian stochastic noise. We observe a smooth transition between a Lorentzian line-profile and a Gaussian one on increasing the incident power or the 


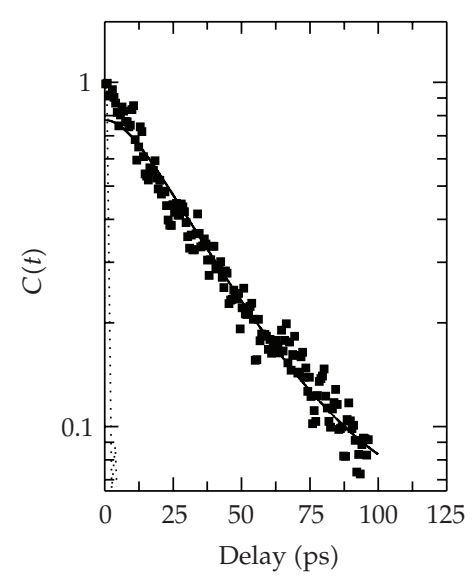

(a)

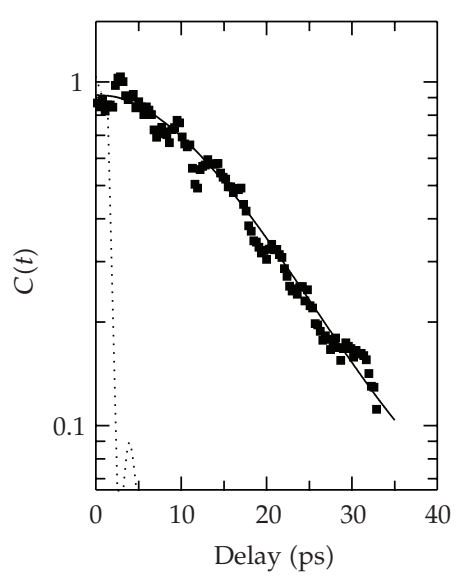

(b)

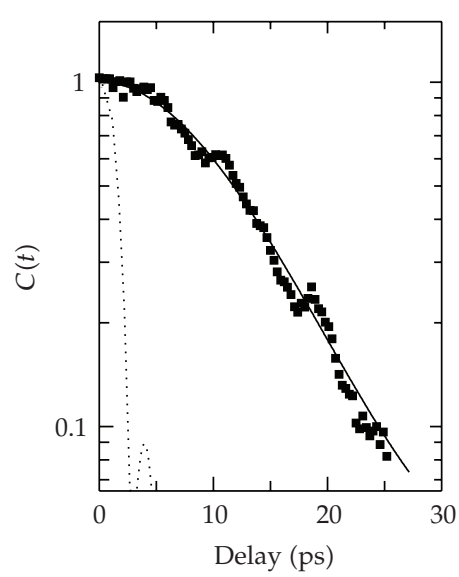

(c)

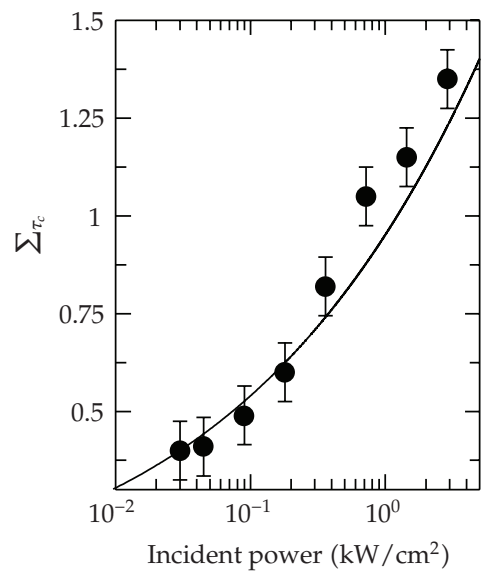

(d)

Figure 6: (a), (b), (c): Interferogram contrast $C(t)$ of the photoluminescence signal of a single InAs/GaAs quantum dot at $10 \mathrm{~K}$, on semilogarithmic plots, for three different incident powers: 0.18 (a), 0.72 (b), and $2.88 \mathrm{~kW} \cdot \mathrm{cm}^{-2}$ (c). Data (squares), system response function (dotted line), theoretical fits (solid line) obtained by the convolution of the system response function with $\phi_{G_{N}}(t)$. (d) Fitting parameter $\Sigma \tau_{c}$ versus incident power. Data (symbols), and calculations (solid lines) are plotted as a function of incident power, on a semilogarithmic scale.

temperature for a QD in a simple heterostructure. We interpret the existence of motional narrowing at low incident power or low temperature as a striking manifestation of the asymmetry of the jump processes in semiconductor QDs.

The sample structure is simpler than in the previous case and it consists of a single QD layer embedded in GaAs so that no electric field is applied to the QD sample. In Figure 6, we display the measured (squares) interference contrast $C(t)$ for the emission spectrum of a single InAs/GaAs quantum dot at $10 \mathrm{~K}$, on semilogarithmic plots, for three different incident powers: $0.18(\mathrm{a}), 0.72(\mathrm{~b})$, and $2.88 \mathrm{~kW} \cdot \mathrm{cm}^{-2}$ (c). We first observe that the coherence relaxation dynamics become faster when increasing the incident power. Moreover, we notice a gradual modification in the shape of $C(t)$. At low power (Figure 6(a)), the interference contrast decay is quasi-exponential with a time constant of $29 \mathrm{ps}$, thus corresponding to a quasi-Lorentzian 
profile with a full width at half maximum of $45 \mu \mathrm{eV}$. At higher power (Figure 6(b)), $C(t)$ has a Gaussian decay at short times $(t \leq 15 \mathrm{ps})$, and remains exponential at longer times. The line has an intermediate profile, and here its full width at half maximum is $105 \mu \mathrm{eV}$. At the highest incident power (Figure 6(c)), the interference contrast decay is predominantly Gaussian, thus corresponding to a quasi-Gaussian profile, with a full width at half maximum of $155 \mu \mathrm{eV}$.

A quantitative interpretation of our measurements is achieved by comparing our experimental data with the convolution of $\phi_{G_{N}}(t)(2.12)$ with the system response function (dotted lines in Figure 6) which is obtained under white light illumination. The calculated fits are displayed in solid line in Figure 6. We obtain an excellent agreement with increasing values of $\Sigma \tau_{c}$ of 0.6 in (a), 1.05 in (b), and 1.35 in (c), where the correlation time $\tau_{c}$ has a constant value of $10 \mathrm{ps}$. In Figure 6(d), we display the whole set of values of $\Sigma \tau_{c}$ as a function of the excitation density, on a semilogarithmic scale. This parameter characterizes the shape of the emission spectrum, and the crossover from the exponential to the Gaussian decoherence dynamics occurs around $\Sigma \tau_{c} \sim 1$. Its gradual increase demonstrates the transition from the fast modulation limit to the slow modulation one when increasing the incident power. As a matter of fact, it shows that motional narrowing occurs for low excitation. We confront the extracted values of $\Sigma \tau_{c}$ with the theoretical values that are calculated with (2.13), and we observe a fair agreement for the set of parameters $\hbar \Sigma_{s} \sim 400 \mu \mathrm{eV}, \tau_{\downarrow} \sim 10 \mathrm{ps}$, and $1 / \tau_{\uparrow}=\left(1 / \tau_{0}\right) \sqrt{P}$, where $\tau_{0} \sim 1.6 \mathrm{~ns}$ and $P$ is in unit of $1 \mathrm{~kW} \cdot \mathrm{cm}^{-2}[11]$.

The asymmetry between the power dependences of $\tau_{\downarrow}$ and $\tau_{\uparrow}$ stems from the existence of different microscopic processes of various efficiencies in semiconductor physics. The constant value of $10 \mathrm{ps}$ for $\tau_{\downarrow}$ is consistent with an optical-phonon assisted capture in the traps around the quantum dot [11]. On the other hand, the inverse process of carrier escape by optical-phonon absorption is strongly inhibited at low temperature, so that it does not significantly contribute to the value of $\tau_{\uparrow}$. In fact, the escape rate dependence on incident power is a characteristic of Auger-type processes. In the elastic collision of two carriers where one is ejected from the trap while a delocalized carrier relaxes in energy, the escape rate is proportional to the density of delocalized carriers, which increases with incident power [11].

As explained above, the asymmetry of the capture and escape mechanisms is in fact the fundamental reason why motional narrowing strikingly occurs when decreasing the incident power or the temperature. If both processes had the same efficiency $\left(\tau_{\uparrow}=\tau_{\downarrow}\right)$, we would have $\tau_{c}=\tau_{\uparrow} / 2$ and $\Sigma=\Sigma_{s}$ which means that the spectral modulation amplitude would not depend on the time constant $\tau_{\uparrow}$. Therefore, the ratio $\Sigma \tau_{c}$ could only decrease when increasing the reservoir excitation. This situation corresponds to the well-known phenomenology from nuclear magnetic resonance where the activation of the nuclei motion induces the motional narrowing effect. In the present case where $\tau_{\downarrow} / \tau_{\uparrow} \leq 10^{-2}$, we are in the opposite regime where the correlation time is merely constant with relative variation smaller than $10^{-2}$, whereas the spectral modulation amplitude shows a steep increase with $\tau_{\downarrow} / \tau_{\uparrow}\left(\Sigma \alpha \sqrt{\tau_{\downarrow} / \tau_{\uparrow}}\right)$. The ratio $\Sigma \tau_{c}$ thus increases when increasing the reservoir excitation. Similar results have been obtained by means of temperature-dependent measurements where the transition from Lorentzian to Gaussian line-profiles occurs on increasing the temperature, thus demonstrating again the unconventional phenomenology of motional narrowing in quantum dots [11].

\section{Conclusion}

In this review, we have presented a general theoretical description of the extrinsic dephasing mechanism of spectral diffusion that dominates the QD decoherence at low temperature. We 
have discussed the limits of random telegraph and Gaussian stochastic noises and shown that the combination of both approaches in the framework of the pre-Gaussian noise theory allows a quantitative interpretation of high-resolution experiments in single semiconductor QDs. We emphasize the generality and the versatility of our model where the inclusion of asymmetric jump processes appears as an essential extension for the understanding of semiconductor QD physics, and hopefully, more generally, for quantum information devices based on solid-state nanostructures.

\section{References}

[1] A. Wallraff, D. I. Schuster, A. Blais, et al., "Strong coupling of a single photon to a superconducting qubit using circuit quantum electrodynamics," Nature, vol. 431, no. 7005, pp. 162-167, 2004.

[2] A. Yu. Kitaev, "Fault-tolerant quantum computation by anyons," Annals of Physics, vol. 303, no. 1, pp. 2-30, 2003.

[3] "Recent advances in quantum dots physics," Special issue of Comptes Rendus Physique, vol. 9, 2008.

[4] P. Michler, A. Kiraz, C. Becher, et al., "A quantum dot single-photon turnstile device," Science, vol. 290, no. 5500, pp. 2282-2285, 2000.

[5] C. Santori, D. Fattal, J. Vučkovic, G. S. Solomon, and Y. Yamamoto, "Indistinguishable photons from a single-photon device," Nature, vol. 419, no. 6907, pp. 594-597, 2002.

[6] X. Li, Y. Wu, D. Steel, et al., "An all-optical quantum gate in a semiconductor quantum dot," Science, vol. 301, no. 5634, pp. 809-811, 2003.

[7] S. A. Empedocles, D. J. Norris, and M. G. Bawendi, "Photoluminescence spectroscopy of single CdSe nanocrystallite quantum dots," Physical Review Letters, vol. 77, no. 18, pp. 3873-3876, 1996.

[8] H. D. Robinson and B. B. Goldberg, "Light-induced spectral diffusion in single self-assembled quantum dots," Physical Review B, vol. 61, no. 8, pp. R5086-R5089, 2000.

[9] V. Türck, S. Rodt, O. Stier, et al., "Effect of random field fluctuations on excitonic transitions of individual CdSe quantum dots," Physical Review B, vol. 61, no. 15, pp. 9944-9947, 2000.

[10] K. Wódkiewicz, B. W. Shore, and J. H. Eberly, "Pre-Gaussian noise in strong laser-atom interactions," Journal of the Optical Society of America B, vol. 1, no. 3, pp. 398-405, 1984.

[11] A. Berthelot, I. Favero, G. Cassabois, et al., "Unconventional motional narrowing in the optical spectrum of a semiconductor quantum dot," Nature Physics, vol. 2, no. 11, pp. 759-764, 2006.

[12] R. Kubo, "A stochastic theory of line-shape and relaxation," in Fluctuation, Relaxation and Resonance in Magnetic Systems, D. Ter Haar, Ed., p. 23, Oliver and Boyd, Edinburgh, UK, 1962.

[13] C. Kammerer, G. Cassabois, C. Voisin, et al., "Interferometric correlation spectroscopy in single quantum dots," Applied Physics Letters, vol. 81, no. 15, p. 2737, 2002.

[14] A. Berthelot, G. Cassabois, C. Voisin, et al., "Voltage-controlled motional narrowing in a semiconductor quantum dot," New Journal of Physics, vol. 11, no. 9, Article ID 093032, 8 pages, 2009.

[15] V. Karpus and V. I. Perel, "Multiphonon ionization of deep centers in semiconductors in an electric field," Sov. JETP, vol. 64, p. 1376, 1986.

[16] S. W. Lin, C. Balocco, M. Missous, A. R. Peaker, and A. M. Song, "Coexistence of deep levels with optically active InAs quantum dots," Physical Review B, vol. 72, no. 16, Article ID 165302, 7 pages, 2005. 


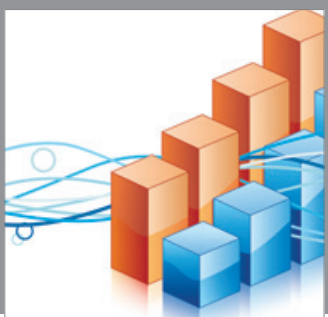

Advances in

Operations Research

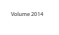

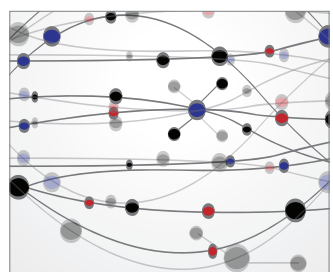

\section{The Scientific} World Journal
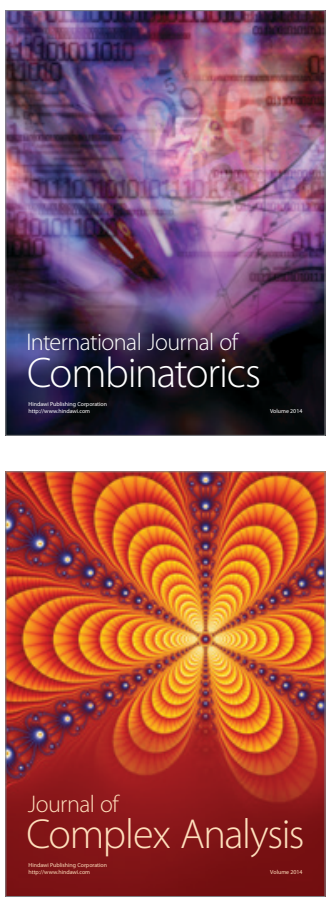

International Journal of

Mathematics and

Mathematical

Sciences
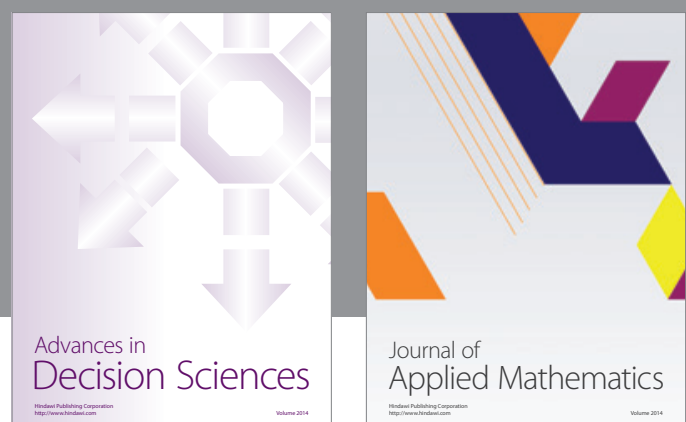

Journal of

Applied Mathematics
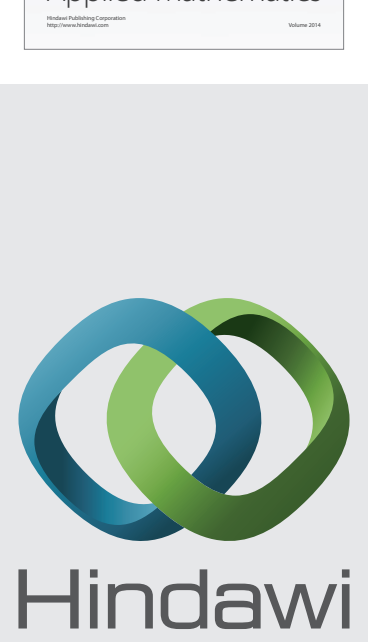

Submit your manuscripts at http://www.hindawi.com
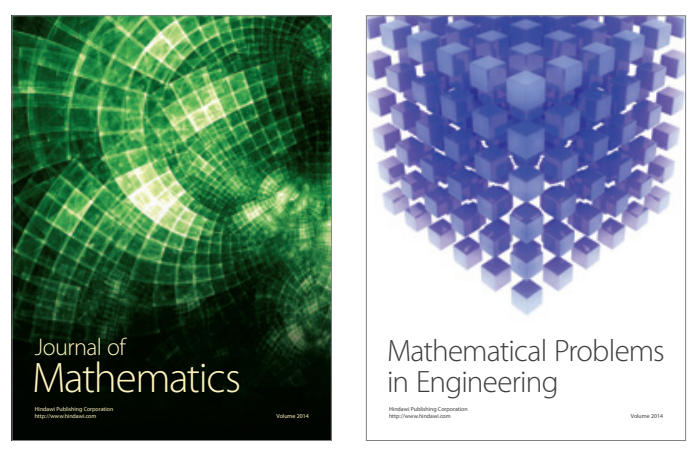

Mathematical Problems in Engineering
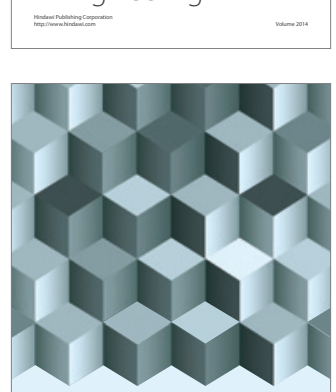

Journal of

Function Spaces
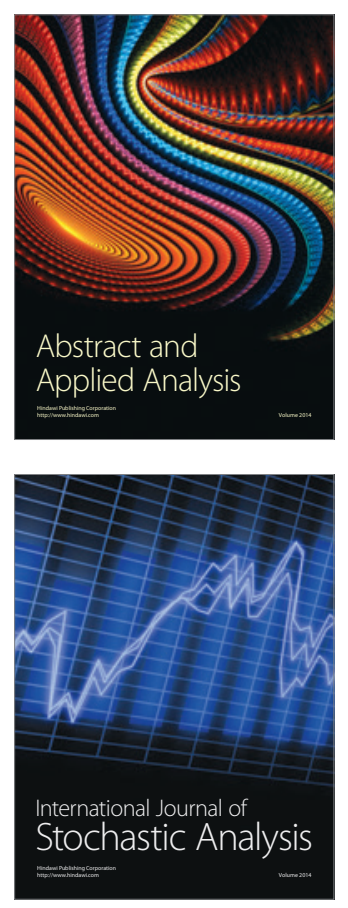

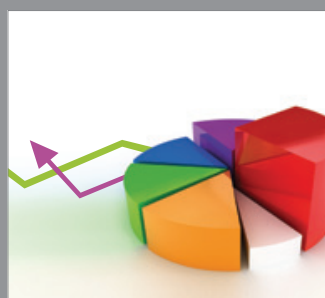

ournal of

Probability and Statistics

Promensencen
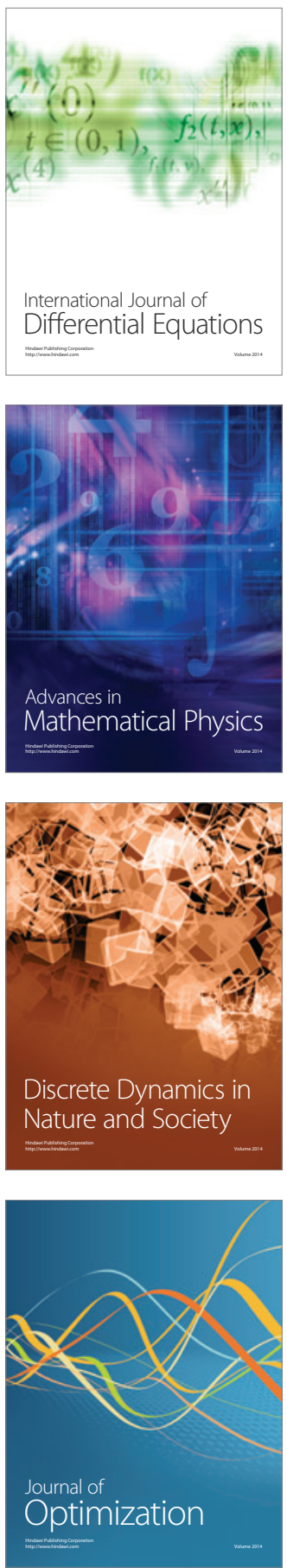